\title{
Motion Planning, Design Optimization and Fabrication of Ferromagnetic Swimmers
}

\author{
Jaskaran Grover, Daniel Vedova, Nalini Jain, Matthew Travers and Howie Choset \\ The Robotics Institute, Carnegie Mellon University, USA \\ Email: (jaskarag, dkv, nalinij, mtravers, choset)@andrew.cmu.edu
}

\begin{abstract}
Small-scale robots have the potential to impact many areas of medicine and manufacturing including targeted drug delivery, telemetry and micromanipulation. This paper develops an algorithmic framework for regulating external magnetic fields to induce motion in millimeter-scale robots in a viscous liquid, to simulate the physics of swimming at the micrometer scale. Our approach for planning motions for these swimmers is based on tools from geometric mechanics that provide a novel means to design periodic changes in the physical shape of a robot that propels it in a desired direction. Using these tools, we are able to derive new motion primitives for generating locomotion in these swimmers. We use these primitives for optimizing swimming efficiency as a function of its internal magnetization and describe a principled approach to encode the best magnetization distributions in the swimmers. We validate this procedure experimentally and conclude by implementing these newly computed motion primitives on several magnetic swimmer prototypes that include two-link and three-link swimmers.
\end{abstract}

\section{INTRODUCTION}

Planning motions for small-scale robots in a liquid can have a huge impact for applications such as microsurgery, telemetry and micromanipulation. Due to the small size of these robots, locomotion at the sub-millimeter scale is dominated by low Reynolds $(R e)$ hydrodynamics. One consequence of swimming in this regime is that motion is highly damped and inertial effects have no contribution towards movement [4]. Therefore, the task of designing robots at this scale requires accounting for the physics of swimming as well as actuation that scales to this regime. The use of on-board actuation is not an option because programmable micromotors that scale to this regime do not exist. Therefore, one solution is to use an external source of actuation such as thermal, chemical or magnetic to wirelessly induce motion in an electrically passive robot. Motivated by this idea, the aim of this paper is to synthesize novel motion primitives for a magnetic robot that responds to magnetic fields, and experimentally fabricate an optimal swimmer for point-to-point steering.

External magnetic fields interact with the intrinsic magnetization distributions (magnetic dipole moments) of these robots by creating a torque or force which causes the robot to bend in response [5, 14]. Therefore, by carefully programming the internal magnetization profiles and prescribing a suitable external field, we demonstrate an algorithmic framework to orchestrate shape changes that generate effective motions in a two-link ferromagnetic swimmer (Fig. 1). Although, it is well known that a two-link robot cannot achieve net displacement in a low $R e$ regime using internally actuated shape changes

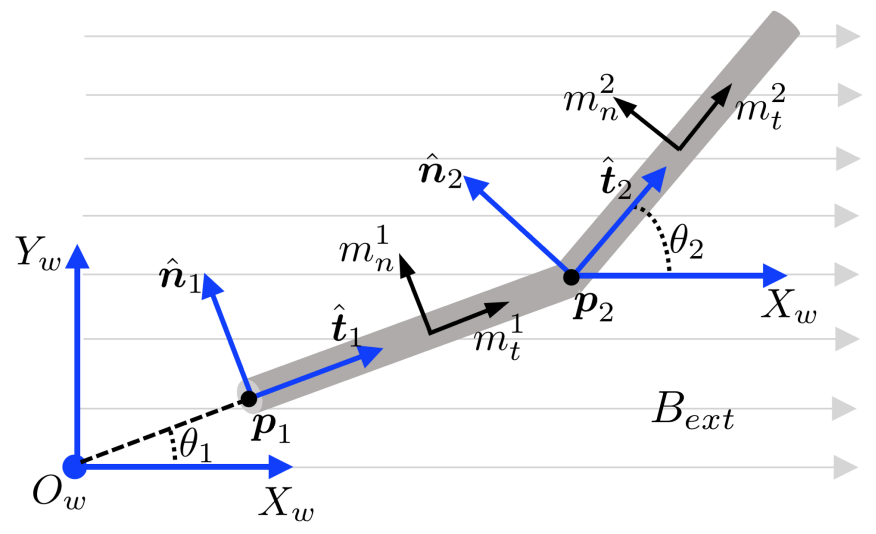

Fig. 1: Schematic of a two-link magnetic swimmer. Gray arrows show external magnetic field, black arrows show internal magnetizations and the joint between the links is springless.

(Scallop theorem [18]), the system we consider escapes this constraint by using external actuation and asymmetric magnetizations which allow it to locomote using reciprocal motions. This work addresses the following technical questions:

1) Given a magnetic swimmer, how do we determine control inputs (i.e. external magnetic field) that induce translation and turning motions in the swimmer ?

2) Given an input with fixed amplitude and frequency, what are the best values of internal magnetizations that optimize the average translational speed of swimmer?

3) Finally, how can these design variables be used to experimentally fabricate an optimal swimmer for locomotion?

As a first step towards addressing these questions, we derive a physics based model for the dynamics of this swimmer by incorporating hydrodynamic and magnetic effects borrowing ideas from previous works [2, 3, 10] (Sec. III). Based on this model, we identify symmetries in the dynamics which allow us to only focus on a representative class of swimmers that are easier to analyze and fabricate (Sec. IV). Next, we address how to compute external field inputs (motion primitives) for these swimmers to induce net locomotion along a desired direction (Sec. V). To that end, we show how gait design tools from geometric mechanics literature can be used to our advantage to prescribe magnetic fields that advance these swimmers in desired directions. Using these tools, we not only motivate previously proposed primitives [11], but also synthesize new 
primitives that have not been explored before. We show that asymptotic stability offered by the existing primitive allows us to synthesize new primitives and compose them to realize turning in place and trajectory following motion (Sec. VI).

Next, we analyze the efficiency of swimming measured using average forward translational speed of the swimmer as a function of its internal magnetization strength (Sec. VII). We numerically solve an optimization problem that identifies the best distribution of internal magnetizations that maximizes the swimming efficiency for a given amplitude of magnetic field.

Although the problem of efficiency optimization is explored in [11], we go a step further and describe a principled approach to experimentally induce these optimal magnetization distributions in millimeter scale ferromagnetic filaments. We finally fabricate these optimal swimmers and validate the motion primitives derived from simulation on these swimmers. Specifically, we show timing test results from fabrication, average speed v/s internal magnetizations trends and swimming trajectories from (1) translation using classical primitives (2) translation using curvature-function based primitive (3) turning-in-place motions and (4) following a rectangle by composing primitives. These results are shown in Sec. VIII for the case of a two link swimmer. We also show experimental results with a three-link swimmer by applying the control inputs for a two-link swimmer to a three-link swimmer.

\section{PRIOR WORK}

Several existing approaches in the literature provide inspiration for the results presented in this work. Of these, two are especially relevant to the results we present. In the first category, we describe existing tools for computing gaits for low $R e$ swimmers and in the following category, we describe existing magnetic swimmers and their modes of propulsion.

\section{A. Geometric motion planning and optimal gait synthesis}

In 1976, Edward Purcell analyzed the locomotion of artificial mechanisms in the low Re regime and established the Scallop theorem [18]. He also proposed the simplest mechanical device: a three-link planar swimmer capable of swimming in this regime by using changes in its internal shape that are non-time reversible. Since his initial work on the internally actuated three-link planar swimmer, much of the subsequent research has focused on planning and computing optimal gaits for the three-link swimmer [20]. In addition to using numerical optimization for computing optimal gaits for these planar systems, authors in [21] use the minimum principle to derive maximum-displacement gaits. Authors in [12] demonstrate that using visual tools derived from geometric analysis, it is possible to synthesize gaits for such a swimmer that make it move along a desired direction in the world. The work in [1] develops analytical techniques to extend gait design to articulated systems with however many links such as a snake like robot locomoting in granular media and a low$R e$ swimmer respectively. Additionally, gait design for threedimensional swimmers has been recently considered in [9] using ideas from geometric mechanics.

\section{B. Magnetic microswimmers}

In contrast to these works that mostly address motion planning for swimmers that are actuated using on board servos, there has also been significant work on inducing propulsion in swimmers using magnetic fields. There are three prominent types of magnetic microswimmers that currently exist. These include a swimmer made with a rigid helical tail which propels with a corkscrew like motion in a rotating magnetic field $[8,17,19]$. The second category consists of swimmers with flexible bodies as considered in $[7,13,16]$. Under the action of an oscillating magnetic field, the flexible body undulates in a non-reciprocal manner ultimately resulting in net propulsion of the swimmer. Some of these swimmers have been analytically modeled in $[6,11]$. The third category consists of articulated swimmers made with discrete links attached to each other which undergo periodic undulations in oscillating magnetic fields. Authors in [14] develop such a swimmer consisting of links connected to each other with flexible hinges. By developing a lumped parameter model of such swimmers, authors in [10] solve a design optimization to determine parameters that maximize displacement and swimming speed.

\section{MATHEMATICAL MODEL}

We now derive a mathematical model for the swimmer that captures the effects of hydrodynamic and magnetic interactions. We will use this model for synthesizing motion plans and computing optimal physical parameters for maximizing swimming efficiency. Our swimmer consists of two millimeterscale slender uniformly magnetized links connected by a passive joint. We assume that the swimmer is fully submerged in a liquid of high viscosity $(\mu)$ such as glycerin to simulate low $R e$ hydrodynamics $(R e=\rho u L / \mu)$. Here $\rho$ is the liquid's density, $u$ is the swimmer's speed and $L$ is the length (for each link). Fig. 1 shows a schematic of this swimmer.

Let $W$ denote the inertial frame. For each link $i$ where $i \in\{1,2\}$, the body frame $\boldsymbol{b}_{i}$ is a frame that is rigidly attached to the link at its vertex. The tangential axis along the body frame is denoted by $\hat{\boldsymbol{t}}_{i}$ and the normal axis of the body frame is denoted by $\hat{\boldsymbol{n}}_{i}$. Thus, it is easy to see that $\hat{\boldsymbol{t}}_{i}=\left(\cos \theta_{i}, \sin \theta_{i}\right)$ and $\hat{\boldsymbol{n}}_{i}=\left(-\sin \theta_{i}, \cos \theta_{i}\right)$ where $\theta_{i}$ is the angle between $\hat{\boldsymbol{t}}_{i}$ and $X_{w}$. In this representation, one can note that $\boldsymbol{b}_{i}=\left\{\left(\hat{\boldsymbol{t}}_{i}, \hat{\boldsymbol{n}}_{i}\right)\right\}$. The configuration space of the swimmer is $Q=S E(2) \times S^{1}$ where the first component $S E(2)$ corresponds to the position and orientation of the body frame of the first link relative to the world. The second component $\left(S^{1}\right)$ corresponds to the orientation of the second link relative to the world. Hence the configuration of the swimmer is identified by $\boldsymbol{q}=\left(x, y, \theta_{1}, \theta_{2}\right)=\left(\boldsymbol{p}_{\mathbf{1}}, \boldsymbol{\theta}\right)$ where $\boldsymbol{p}_{\mathbf{1}}=(x, y)$ and $\boldsymbol{\theta}=\left(\theta_{1}, \theta_{2}\right)$. We now describe magnetic properties of the links. Each link is assumed to be ferromagnetic which means that it has a permanent magnetic dipole moment in its volume. Magnetization of a link is defined relative to its body frame $\boldsymbol{b}_{i}$ as shown in Fig. 1. Here, $m_{t}^{i}$ and $m_{n}^{i}$ are dimensionless numbers that quantify the strengths of magnetization along $\hat{\boldsymbol{t}}_{i}$ and $\hat{\boldsymbol{n}}_{i}$ of link $i$ respectively. Let $h>0$ denote the value 
of internal magnetization expressed in Am units. Relative to inertial frame $W$, link $i$ 's magnetization is expressed as

$$
\begin{aligned}
\boldsymbol{M}^{i} & =\left(m_{t}^{i} \hat{\boldsymbol{t}}_{i}+m_{n}^{i} \hat{\boldsymbol{n}}_{i}\right) h \\
& =\left(m_{t}^{i} \cos \theta_{i}-m_{n}^{i} \sin \theta_{i}, m_{t}^{i} \sin \theta_{i}+m_{n}^{i} \cos \theta_{i}\right) h
\end{aligned}
$$

For a link with length $L$ and internal magnetization $M^{i}$, the external magnetic field applies a torque on the link given by $\boldsymbol{\tau}_{i, m}=L \boldsymbol{M}^{i} \times \boldsymbol{B}(t)$ where $\boldsymbol{B}(t)=\left(B_{x}(t), B_{y}(t)\right)$ denotes the $X_{w}$ and $Y_{w}$ components of the external magnetic field measured in Tesla/Gauss. We also assume that there is no torsional spring connecting the two links. This is because driftlessness of the dynamics will be necessary for synthesis of the motion plans in Sec. V. The model of the swimmer is derived by incorporating forces and torques from hydrodynamic and magnetic effects as follows:

1) $\boldsymbol{F}_{i, h}:$ Hydrodynamic drag force on link $i$ in world frame

2) $\boldsymbol{\tau}_{i, h}^{\boldsymbol{p}_{m}}$ : Hydrodynamic drag torque on link $i$ about the $Z_{w}$ axis passing through $\boldsymbol{p}_{m}$ expressed in world frame

3) $\tau_{i, m}$ : Magnetic torque on link $i$ expressed in world frame where $(i, m \in\{1,2\})$ denote link index and point index respectively. From the assumptions of resistive force theory, it is known that the hydrodynamic forces and torques on a link are linear in the velocity of the link. The exact expressions for these forces can be found in [1]. Additionally, the net force and moment on a system in quasistatic equilibrium vanishes:

$$
\begin{aligned}
\boldsymbol{F}_{1, h}+\boldsymbol{F}_{2, h} & =0 \\
\boldsymbol{\tau}_{1, h}^{\boldsymbol{p}_{1}}+\boldsymbol{\tau}_{2, h}^{\boldsymbol{p}_{1}}+\boldsymbol{\tau}_{1, m}+\boldsymbol{\tau}_{2, m} & =0 \\
\boldsymbol{\tau}_{2, h}^{\boldsymbol{p}_{2}}+\boldsymbol{\tau}_{2, m} & =0
\end{aligned}
$$

After substituting the expressions for the forces and torques ([1]) and rearranging, we can rewrite Eqs. $2 a-2 c$ in the form of a control affine system. The control input to the system is defined by the spatial magnetic fields i.e. $\boldsymbol{u}=\left(B_{x}(t), B_{y}(t)\right)$ and the state of the system is $\boldsymbol{q}(t)$.

$$
\begin{aligned}
\dot{\boldsymbol{q}} & =\boldsymbol{g}_{1}(\boldsymbol{q}) B_{x}(t)+\boldsymbol{g}_{2}(\boldsymbol{q}) B_{y}(t) \\
& =\boldsymbol{G}(\boldsymbol{q}) \boldsymbol{u}
\end{aligned}
$$

We define a vector $\boldsymbol{m}=\left(m_{t}^{1}, m_{t}^{2}, m_{n}^{1}, m_{n}^{2}\right)$ that denotes the internal magnetizations of the links (the subscript indicates tangential/normal while the superscript indicates the link index). To emphasize on the dependence of the matrix $\boldsymbol{G}(\boldsymbol{q})$ on $\boldsymbol{m}$, we will explicitly denote it as $\boldsymbol{G}(\boldsymbol{q}, \boldsymbol{m})$.

\section{SYMMETRIES IN DYNAMICS}

In the derivation of the dynamics model, we assumed that each link of the swimmer has magnetization components along the link's tangential (i.e. $m_{t}^{i} \neq 0$ ) and normal directions (i.e. $m_{n}^{i} \neq 0$ ). However, intuitively it should be possible to modify the external field in a way such that it exerts the same torque on each link as if it had internal magnetizations only along its tangential direction. Swimmers with tangential only magnetizations are more intuitive to analyze since we can interpret their links as slender bar magnets. Additionally, as we will show, requiring only tangential magnetizations gives

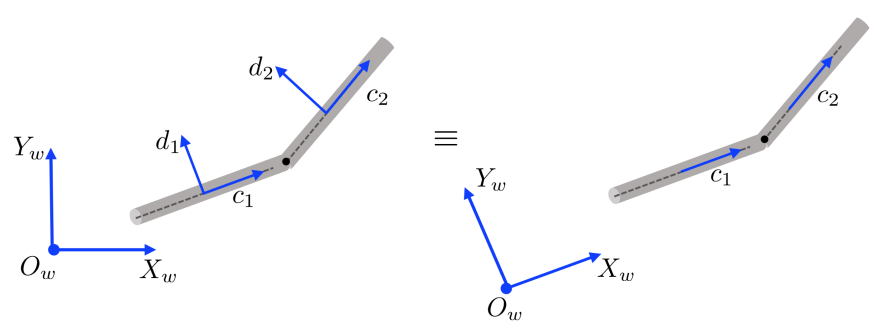

Fig. 2: Equivalence between swimmers with tangential+normal magnetizations and only tangential magnetizations

us a representative class of swimmers which have the same locomotive functionalities as swimmers with both components of magnetizations. We state this symmetry theorem as follows:

Theorem 4.1: Any trajectory of a two link swimmer with both normal and tangential magnetizations and a given magnetic field can be obtained from a two link swimmer with only tangential magnetizations and a transformed magnetic field

Proof: We begin by establishing some notation. We will use the superscript tan + nor to refer to a swimmer with both tangential and normal magnetization components. Likewise, we will use the superscript tan to refer to a swimmer with only tangential magnetization components. Recall that the differential equations governing the dynamics of a two link swimmer are (Eq. 3) $\dot{\boldsymbol{q}}=G(\boldsymbol{q}, \boldsymbol{m}) \boldsymbol{u}(t)$ where $\boldsymbol{m}=\left(m_{t}^{1}, m_{t}^{2}, m_{n}^{1}, m_{n}^{2}\right)$ are the magnetization components and $\boldsymbol{u}(t)=\left(B_{x}(t), B_{y}(t)\right)$ is the magnetic field. Let $\boldsymbol{q}_{\text {tan }+ \text { nor }}(t)$ be the solution to Eq. 3 for $\boldsymbol{m}=\boldsymbol{m}^{\text {tan }+ \text { nor }}=$ $\left(c_{1}, c_{2}, d_{1}, d_{2}\right)$ for $c_{1}, c_{2}, d_{1}, d_{2} \in \mathbb{R}-\{0\}$ such that $\frac{c_{1}}{c_{2}}=$ $\frac{d_{1}}{d_{2}}, \boldsymbol{q}(0)=0$ and $\boldsymbol{u}(t)=\boldsymbol{u}^{\text {tan+nor }}(t)=\left(B_{x}(t), B_{y}(t)\right)$ (Fig. 2 left) Similarly, let $\boldsymbol{q}_{\text {tan }}(t)$ be solution to Eq. 3 for $\boldsymbol{m}=\boldsymbol{m}^{\boldsymbol{t a n}}=\left(c_{1}, c_{2}, 0,0\right), \boldsymbol{q}(0)=0$ and $\boldsymbol{u}(t)=\boldsymbol{u}^{\boldsymbol{t a n}}(t)=$ $\left(B_{x}(t)+\frac{d_{1}}{c_{1}} B_{y}(t),-\frac{d_{1}}{c_{1}} B_{x}(t)+B_{y}(t)\right)$ (Fig. 2 right).

Then for this choice of external magnetic field and magnetizations, we will show that $\boldsymbol{q}_{\text {tan }+ \text { nor }}(t)=\boldsymbol{q}_{\text {tan }}(t) \forall t \geq 0$. We know that the dynamics of the swimmer are governed by the matrix $G(\boldsymbol{q}, \boldsymbol{m}) \boldsymbol{u}$ which depends on magnetic torques $\boldsymbol{\tau}_{\boldsymbol{i}, \boldsymbol{m}}$ aside from hydrodynamic forces and torques. We now show that magnetic torques on these two types of swimmers (i.e. Fig. 2 left and Fig. 2 right) are identical for the given choice of magnetizations and external magnetic fields. For the swimmer with both tangential and normal components (Fig.2 left), note:

$$
\begin{aligned}
\tau_{\boldsymbol{i}, \boldsymbol{m}}^{\text {tan }+ \text { nor }} & =\left(c_{i} \hat{\boldsymbol{t}}_{i}+d_{i} \hat{\boldsymbol{n}}_{i}\right) \times \boldsymbol{u}^{\text {tan }+ \text { nor }}(t) \\
& =\left(c_{i} I \hat{\boldsymbol{t}}_{i}+d_{i} R_{\frac{\pi}{2}} \hat{\boldsymbol{t}}_{i}\right) \times \boldsymbol{u}^{\text {tan }+ \text { nor }}(t) \\
& =\left(c_{i} I+d_{i} R_{\frac{\pi}{2}}\right) \hat{\boldsymbol{t}}_{i} \times \boldsymbol{u}^{\text {tan }+ \text { nor }}(t)
\end{aligned}
$$

Note from the definition of the two controls that

$$
\boldsymbol{u}^{\tan }(t)=\left(I-\frac{d_{1}}{c_{1}} R_{\frac{\pi}{2}}\right) \boldsymbol{u}^{\text {tan }+ \text { nor }}(t)
$$

where $I$ is the identity matrix. For the swimmer with only 
tangential magnetizations (Fig.2 right), note that

$$
\begin{aligned}
\boldsymbol{\tau}_{\boldsymbol{i}, \boldsymbol{m}}^{\tan }(t) & =c_{i} \hat{\boldsymbol{t}}_{i} \times \boldsymbol{u}^{\tan }(t) \\
& =c_{i} \hat{\boldsymbol{t}}_{i} \times\left(I-\frac{d_{1}}{c_{1}} R_{\frac{\pi}{2}}\right) \boldsymbol{u}^{\text {tan }+ \text { nor }}(t) \\
& =c_{i} \hat{\boldsymbol{t}}_{i} \times \boldsymbol{u}^{\text {tan }+ \text { nor }}(t)-d_{i} \hat{\boldsymbol{t}}_{i} \times R_{\frac{\pi}{2}} \boldsymbol{u}^{\text {tan }+ \text { nor }}(t) \\
& =c_{i} I \hat{\boldsymbol{t}}_{i} \times \boldsymbol{u}^{\text {tan }+ \text { nor }}(t)+d_{i} R_{\frac{\pi}{2}} \hat{\boldsymbol{t}}_{i} \times \boldsymbol{u}^{\text {tan }+ \text { nor }}(t) \\
& =\left(c_{i} I+d_{i} R_{\frac{\pi}{2}}\right) \hat{\boldsymbol{t}}_{i} \times \boldsymbol{u}^{\text {tan }+ \text { nor }}(t) \\
& =\boldsymbol{\tau}_{\boldsymbol{i}, \boldsymbol{m}}^{\text {tan }}+\boldsymbol{n o r}(t)
\end{aligned}
$$

Therefore, for the given choice of internal magnetizations and external fields, the two swimmers (Fig. 2 left and Fig. 2 right) experience same magnetic torques. Additionally, since the hydrodynamic torques on these systems do not depend on their magnetic properties, both swimmers experience identical hydrodynamic effects as well, i.e. $\boldsymbol{F}_{\boldsymbol{i}, \boldsymbol{h}}^{\boldsymbol{t a n}}=\boldsymbol{F}_{\boldsymbol{i}, \boldsymbol{h}}^{\boldsymbol{t a n}+\boldsymbol{n} \text { or }}$ and $\tau_{i, h}^{t a n}=\tau_{i, h}^{t a n+n o r}$. Hence, the dynamics of these swimmers are identical i.e $G\left(\boldsymbol{q}, \boldsymbol{m}^{\text {tan+nor }}\right) \boldsymbol{u}^{\text {tan+nor }}=$ $G\left(\boldsymbol{q}, \boldsymbol{m}^{\tan }\right) \boldsymbol{u}^{\text {tan }}$ based on Eq. 2. Since the initial condition in both systems is same, the governing IVP for both systems is the same, therefore $\boldsymbol{q}_{\text {tan }+ \text { nor }}(t)=\boldsymbol{q}_{\text {tan }}(t) \forall t \geq 0$.

Therefore, for forgoing discussion, we will only consider swimmers with tangential internal magnetizations components in each link. Let $m_{t}^{1}=c_{1}>0$ and $m_{t}^{2}=c_{2}>0$ denote the strengths of magnetizations in links 1 and 2 respectively. We will assume that $c_{2} \neq c_{1}$ to avoid front back symmetry.

\section{Motion Planning Using Geometric Mechanics}

In Sec III and IV, we have formulated the equations of motion for a two-link swimmer and identified symmetries allowing us to focus only on a simplified swimmer. Based on the model, we can explore the behavior of the swimmer's motion as a function of different types of control inputs $\boldsymbol{u}(t)=\left(B_{x}(t), B_{y}(t)\right)$. In this section, we will demonstrate that by using tools from geometric mechanics we can generate new motion primitives for swimming compared to the ones proposed before. We assumed that the swimmer is springless so there is no drift vector field in the dynamics of the swimmer:

$$
\dot{\boldsymbol{q}}=\boldsymbol{G}(\boldsymbol{q}, \boldsymbol{m}) \boldsymbol{u}
$$

where $\boldsymbol{q}=(\boldsymbol{p}, \boldsymbol{\theta}), \boldsymbol{G}(\boldsymbol{q}, \boldsymbol{m})=\left[\boldsymbol{g}_{1}(\boldsymbol{q}, \boldsymbol{m}), \boldsymbol{g}_{2}(\boldsymbol{q}, \boldsymbol{m})\right]$ and $\boldsymbol{u}(t)=\left(B_{x}(t), B_{y}(t)\right)$. Since the magnetic field is spatially uniform, the instantaneous $(x, y)$ position coordinates of the swimmer do not effect its motion. The only state variables that influence the dynamics are the orientation of the swimmer's links in the inertial frame. Therefore, the dynamics of the position variables i.e. $\dot{\boldsymbol{p}}=(\dot{x}, \dot{y})$ depend exclusively on the orientation variables $\boldsymbol{\theta}=\left(\theta_{1}, \theta_{2}\right)$. Similarly, the dynamics of the orientation variables $\dot{\boldsymbol{\theta}}$ depend exclusively on $\boldsymbol{\theta}$. Hence, we can break Eq. 7 into two separate sub-systems as follows:

$$
\begin{aligned}
\dot{\boldsymbol{q}} & =\boldsymbol{G}(\boldsymbol{q}, \boldsymbol{m}) \boldsymbol{u}=\boldsymbol{G}(\boldsymbol{\theta}, \boldsymbol{m}) \boldsymbol{u} \\
\Longrightarrow \dot{\boldsymbol{p}} & =\boldsymbol{P}(\boldsymbol{\theta}, \boldsymbol{m}) \boldsymbol{u} \\
\dot{\boldsymbol{\theta}} & =\boldsymbol{H}(\boldsymbol{\theta}, \boldsymbol{m}) \boldsymbol{u}
\end{aligned}
$$

where $\boldsymbol{P}(\boldsymbol{\theta}, \boldsymbol{m}) \in \mathbb{R}^{2 \times 2}$ and $\boldsymbol{H}(\boldsymbol{\theta}, \boldsymbol{m}) \in \mathbb{R}^{2 \times 2}$. The exact expressions for these matrices are omitted in the interest of space. Assuming $\boldsymbol{H}(\boldsymbol{\theta}, \boldsymbol{m})$ is invertible on $[-2 \pi, 2 \pi] \times[-2 \pi, 2 \pi]$, we can compute $\boldsymbol{u}$ from Eq. 9 and substitute in Eq. 8 as follows:

$$
\begin{aligned}
\boldsymbol{u} & =\boldsymbol{H}^{-1}(\boldsymbol{\theta}, \boldsymbol{m}) \dot{\boldsymbol{\theta}} \\
\Longrightarrow \dot{\boldsymbol{p}} & =\boldsymbol{P}(\boldsymbol{\theta}, \boldsymbol{m}) \boldsymbol{H}^{-1}(\boldsymbol{\theta}, \boldsymbol{m}) \dot{\boldsymbol{\theta}} \\
\Longrightarrow \dot{\boldsymbol{p}} & =\boldsymbol{J}(\boldsymbol{\theta}, \boldsymbol{m}) \dot{\boldsymbol{\theta}}
\end{aligned}
$$

where $\boldsymbol{J}(\boldsymbol{\theta}, \boldsymbol{m})=\boldsymbol{P}(\boldsymbol{\theta}, \boldsymbol{m}) \boldsymbol{H}^{-1}(\boldsymbol{\theta}, \boldsymbol{m})$. Note that Eq. 10c is in a form similar to the Kinematic Reconstruction Equation

$$
\boldsymbol{\xi}=-A(\boldsymbol{\alpha}) \dot{\boldsymbol{\alpha}}
$$

where $A(\boldsymbol{\alpha}) \in \mathbb{R}^{3 \times 2}$ is known as the local form of a connection. It maps shape velocities to body velocities: $A(\boldsymbol{\alpha})$ : $T_{\alpha_{1}} S^{1} \times T_{\alpha_{2}} S^{1} \longrightarrow \mathfrak{s e}(2)$. In the literature on geometric theory of swimming [15], this equation has been used to synthesize motion primitives for swimmers that are internally actuated i.e. where it is possible to command any values of $\boldsymbol{\alpha}(t)$. On the contrary, note that in Eq. 10c the variables $\left(\theta_{1}, \theta_{2}\right)$ refer to the orientation of the swimmer relative to the world. Additionally, the left hand side of Eq. 10c also involves velocities referenced relative to the inertial frame as opposed to the body velocities $\boldsymbol{\xi}$ expressed in the body frame. Hence, we cannot model this system with a principal fiber bundle structure. Nevertheless, assuming for the moment that we can fully control $\left(\theta_{1}, \theta_{2}\right)$, it is possible to compute the total displacement over a cyclic change in $\left(\theta_{1}, \theta_{2}\right)$ as follows

$$
\begin{aligned}
\boldsymbol{p}(T) & =\int_{0}^{T} \boldsymbol{J}(\boldsymbol{\theta}(t), \boldsymbol{m}) \dot{\boldsymbol{\theta}}(t) d t \\
& =\int_{\gamma} \boldsymbol{J}(\boldsymbol{\theta}, \boldsymbol{m}) d \boldsymbol{\theta} \\
& =\iint_{\mathcal{S}} \nabla \times \boldsymbol{J} d \theta_{1} d \theta_{2} .
\end{aligned}
$$

In Eq. 12, we have used Stokes' theorem ${ }^{1}$ to simplify the problem of computing line integral of the rows of $\boldsymbol{J}(\boldsymbol{\theta}, \boldsymbol{m})$ along $\gamma$, to computing volume integrals of the curvature of $\boldsymbol{J}$ (curl $\boldsymbol{J}$ ) defined over $\mathcal{S}$. We plot the $x$ and $y$ components of curl $\boldsymbol{J}$ in Fig. 3. To synthesize a motion primitive for translation in the $X_{w}$ direction of the world frame, we visually query and inspect regions in Fig. 3 in the orientation angle phase space which enclose a net non-zero volume in $x$ component and zero volume in the $y$ component. Using this visual inspection, we pick a loop in the $\left(\theta_{1}, \theta_{2}\right)$ space that encloses such a region. One such candidate loop is highlighted in red in Fig. 3 and is parametrized as:

$$
\begin{aligned}
& \theta_{1}^{d}(t)=0.35 \sin (\omega t-1.817) \\
& \theta_{2}^{d}(t)=0.53 \sin (\omega t-0.7186)
\end{aligned}
$$

\footnotetext{
${ }^{1}$ Differently from the work in [2], we use Stokes' theorem for magnetic swimmers without springs and visualize the effect of the limit cycles over the full orientation space using curvature function plots in Fig. 3. Secondly, we do not require a small-angle approximation and instead illustrate the effect of the full swing limit cycle on displacement directly.
} 

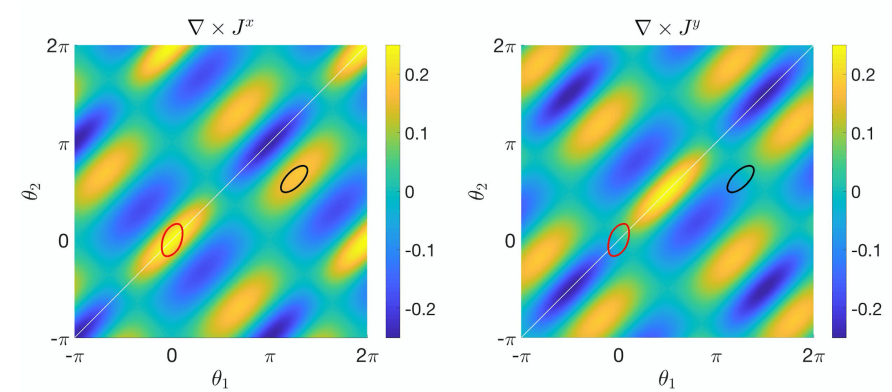

Fig. 3: curl $\boldsymbol{J}$ computed over $[-\pi, 2 \pi] \times[-\pi, 2 \pi]$
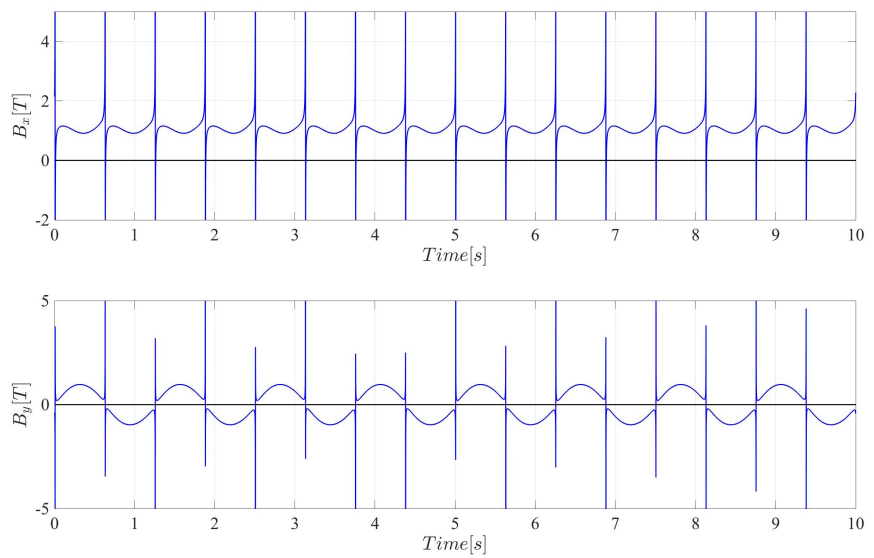

Fig. 4: Magnetic field input for the red loop using Eq. 14

With this parametrization and Eq. 10a, we compute the control:

$$
\boldsymbol{u}(t)=\boldsymbol{H}^{-1}\left(\theta_{1}^{d}(t), \theta_{2}^{d}(t)\right)\left[\begin{array}{c}
\dot{\theta_{1}^{d}} \\
\dot{\theta_{2}^{d}}
\end{array}\right]
$$

The resulting input is plotted in Fig. 4.

\section{A. Relation to previously proposed motion primitive}

Note that the magnetic field input in Fig. 4 exhibits a discontinuity which happens whenever $\theta_{1}(t)=\theta_{2}(t)$. This singularity is the result of the swimmer admitting an instantaneous straightened configuration and is not a locked singularity. We low-pass filter these inputs to remove the singularity and normalize them by their amplitude. This gives

$$
B_{x}(t)=1, B_{y}(t)=\sin \omega t
$$

This control input matches exactly with the ones proposed in $[2,10]$. Using this, we numerically simulate the dynamics of the system Eq. 3 and plot the resulting trajectory of the swimmer in Fig. 5. Note that the swimmer indeed undergoes translation along $X_{w}$ axis as we set out our initial goal.

\section{B. Novel motion primitives}

We extend this tool to synthesize new control inputs that also result in translation along $X_{w}$. To that end, consider a

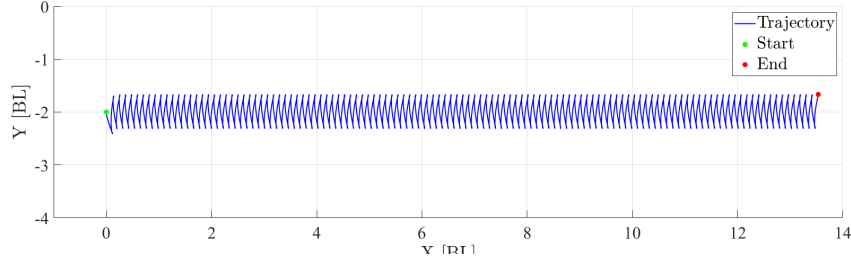

Fig. 5: Translation trajectory using $B_{x}=1, B_{y}=\sin \omega t$

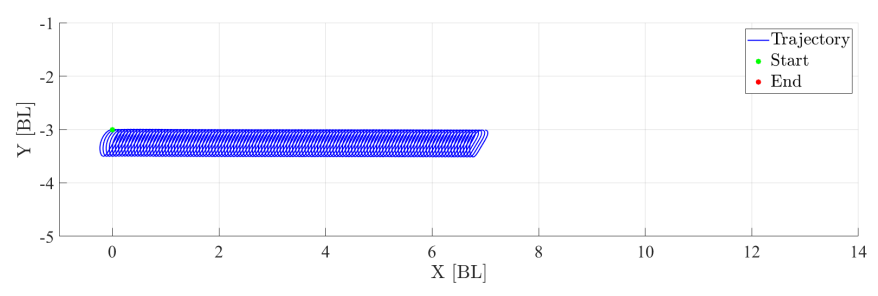

Fig. 6: Translation trajectory using inputs in Eq. 17

time parametrized loop in the $\left(\theta_{1}, \theta_{2}\right)$ defined below

$$
\begin{aligned}
& \theta_{1}^{d}(t)=0.5 \cos \omega t \cos \frac{\pi}{4}-0.25 \sin \omega t \sin \frac{\pi}{4}+\frac{5 \pi}{4} \\
& \theta_{2}^{d}(t)=0.5 \cos \omega t \sin \frac{\pi}{4}+0.25 \sin \omega t \cos \frac{\pi}{4}+\frac{3 \pi}{4}
\end{aligned}
$$

This loop is depicted in black in Fig. 3. Using this parametrization, we compute $\boldsymbol{u}(t)=\left(B_{x}(t), B_{y}(t)\right)$ using Eq. 14. Using interpolation, we write the corresponding control input as:

$$
B_{x}(t)=\sum_{i=1}^{4} a_{i}^{x} \sin \left(b_{i}^{x} t+c_{i}^{x}\right), B_{y}(t)=\sum_{i=1}^{4} a_{i}^{y} \sin \left(b_{i}^{y} t+c_{i}^{y}\right)
$$

To save space, we omit specifying the exact values of $\left\{a_{i}^{x}, b_{i}^{x}, c_{i}^{x}, a_{i}^{y}, b_{i}^{y}, c_{i}^{y}\right\}$. We simulate the system dynamics using this input and show the resulting displacement of the swimmer in Fig. 6. Note from Fig. 6 that the translation distance of the swimmer computed over 15 cycles of executing control Eq. 17 is $\sim 7$ body lengths. However, exciting the swimmer with 15 cycles of the control from Eq. 15 results in a translation of $\sim 14$ body lengths as shown in Fig. 5. This suggests that as measured using average swimming speed, the motion generated by Eq. 15 is two times more efficient than the motion generated by the control in Eq. 14. We validate these primitives by implementing them on our fabricated robots and show in Sec. VIII that this relative efficiency ratio is indeed observed in experiments as well. Next, we will analyze the stability properties of the primitives in Eq. 15 and 17.

\section{Limit CyCle StabiLity}

From simulations, we observe that the steady state motion of the swimmer using the input from Eq. 15 is independent of the swimmer's initial orientation in the world. On the other hand, corresponding to Eq. 17, the steady state response of the swimmer indeed is sensitive to the initial orientation of the links relative to the world. This observation suggests that corresponding to input $\boldsymbol{u}(t)=(1, \sin \omega t)$, the asymptotic trajectory in the $\left(\theta_{1}, \theta_{2}\right)$ phase portrait is locally asymptotically 


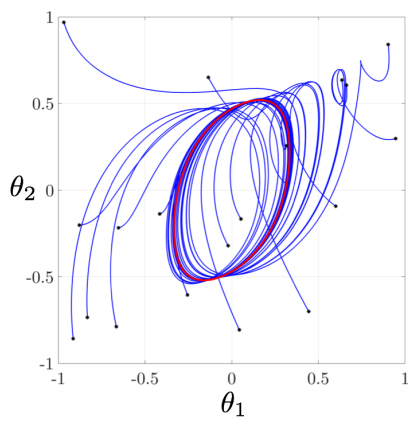

Fig. 7: Limit cycle (red) from different initial orientations

stable with respect to variations in the initial orientation of the swimmer. We state this result in the theorem below:

Theorem 6.1: Given the control input $\boldsymbol{u}(t)=(1, \sin \omega t)$, the flows of the differential equation $\dot{\boldsymbol{\theta}}=\boldsymbol{H}(\boldsymbol{\theta}) \boldsymbol{u}(t)$, $\boldsymbol{\theta}(0)=\left(\theta_{1}(0), \theta_{2}(0)\right)$ converge to the same solution $\boldsymbol{\theta}^{\infty}(t)=$ $\left(\theta_{1}^{\infty}(t), \theta_{2}^{\infty}(t)\right)$ for all $\boldsymbol{\theta}(0)$ close to $\boldsymbol{\theta}^{\infty}(t)$ i.e. the periodic trajectory specified by $\boldsymbol{\theta}^{\infty}(t)$ is locally asymptotically stable.

Proof: See supplementary material

To illustrate this, we plot the flows of the orientation components of $\boldsymbol{q}(t)$ starting from different initial conditions in Fig. 7 using $\boldsymbol{u}(t)=(1, \sin \omega t)$. This figure shows that all these flows converge to the same limit cycle $\left(\theta_{1}^{\infty}(t), \theta_{2}^{\infty}(t)\right)$ (highlighted in red). Moreover, it also illustrates that the limit cycle is not only locally asymptotically stable but that it also has a large basin of attraction. This suggests that it is possible to align the swimmer with the direction of the external magnetic field vector even when the initial orientation of individual links of the swimmer is off by as much as $\pi$ radians relative to the direction the external field. Hence, by switching the direction of the field vector, we can make the swimmer turn-in-place or follow a rectangular trajectory in the workspace. In the interest of space, we skip simulation results and demonstrate experimentally in Fig. 9 (two-link) and Fig. 10 (three-link).

All these derivations have been done for a two-link swimmer because exact inversion in Eq. 10a is only possible for two inputs i.e. $\left(B_{x}(t), B_{y}(t)\right)$ and two outputs i.e. $\left(\theta_{1}, \theta_{2}\right)$. Since a three link swimmer has two inputs and three outputs $\left(\theta_{1}, \theta_{2}, \theta_{3}\right)$, exact inversion in Eq. 10a and control synthesis is not possible. Therefore, for simulations and experiments, we directly used the control inputs for the two link system and applied them on the three link system to study its response.

\section{DESIGN OPTIMIZATION AND FABRICATION}

Using stability analysis, we have demonstrated that the input from Eq. 15 is suitable for point to point steering and turning in place type motions. Given a fixed amplitude and frequency of this input, we would now like to determine parameters intrinsic to the swimmer that maximize its forward translational speed. One such parameter is the intrinsic magnetization of each link which dictates how much magnetic torque that link experiences under the action of a magnetic field. This in-turn regulates the amplitude of undulation and hence the speed. So, we select magnetization as a design variable for optimizing swimming efficiency for a given field amplitude.

\section{A. Optimizing over internal magnetization}

We can either consider optimizing over magnetizations of both links or optimizing over the magnetization of link 2 (head link) for a fixed magnetization of link 1 (tail link). However, note that by increasing the amplitude of external magnetic field, we can realize a proportionate increase in the magnetization of both links. Therefore, we will only optimize over magnetization of link 2 for a fixed magnetization of link 1 i.e. $m_{t}^{1}=c_{1}=1, m_{t}^{2}=c_{2} \neq 0, m_{n}^{1}=m_{n}^{2}=0$. We now pose an optimization problem to compute the values of $c_{2}$ that maximize the total displacement of the swimmer in a fixed duration from $t=0$ to $t=T$. We set $f=0.8 \mathrm{~Hz}$ same as the frequency of actuation in our experimental setup.

$$
\begin{array}{ll}
\underset{c_{2}}{\operatorname{maximize}} & |x(T) / T| \\
\text { subject to } & \dot{\boldsymbol{q}}=\boldsymbol{G}(\boldsymbol{q}, \boldsymbol{m}) \boldsymbol{u} \\
& \boldsymbol{u}(t)=(1, \sin 2 \pi f t), \boldsymbol{q}(0)=\mathbf{0}
\end{array}
$$

We use numerical simulations to solve this optimization problem. Fig. 8a depicts the relation between the absolute average speed of the swimmer for several values of the magnetization of link 2. As we can note, increasing the values of the magnetization of link 2 relative to link 1 results in increasing values of the speed of the swimmer. This is because by increasing strength of magnetization of link 2, it bends with greater amplitude, due to which it drags through more fluid and advances a greater distance forward. The peak from $M \in(0,1)$ exists because the magnetization of link 2 is weak compared to link 1 so the swimmer advances backward. Note that the $X$ axis in Fig. 8a is limited to 6 because the ratio of the strongest to weakest magnetizing field we can generate with our experimental setup (to be described next) is precisely 6 . We conducted a similar optimization for three-link swimmer and determined the optimal magnetization ratio to be 1:4:4.

\section{B. Experimental Fabrication}

We have seen that by increasing the magnetization of the head link relative to the tail link, the efficiency of swimming can be improved. We now describe an experimental procedure to fabricate a two link swimmer with a high value of interlink magnetization ratio. For our experiments, we manufacture ferromagnetic links that are connected with an elastomer to make the two link swimmer. Each link is made by mixing equal parts of unmagnetized ferromagnetic particles Magnequench MQFP-B $(D 50=15 \mu \mathrm{m})$ and resin (Smooth-Cast 326). After curing, the substrate is laser cut into $6.5 \mathrm{~mm} \times 4.2 \mathrm{~mm} \times 1.75$ $\mathrm{mm}$. rectangular links. To magnetize a link, we use a pair of neodymium magnets that generate strong magnetizing fields in $0.1 \mathrm{~T}-0.6 \mathrm{~T}$ in a height range of 0 to $3.5 \mathrm{cms}$ (supplementary material). Each link is suspended between these magnets which induces magnetic moments along its length . We then join two such links with an elastomer of low-bending stiffness to make the complete swimmer. We assume that the strength of magnetization in a link $M_{\text {induced }} \propto B_{\text {magnetizing }}$ between 


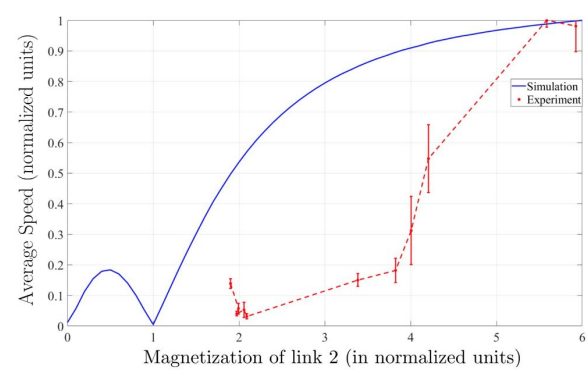

(a) Average speed v/s link 2 magnetization.

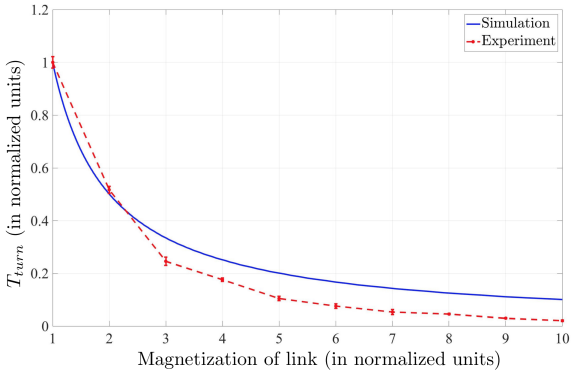

(b) Turning time v/s link magnetization.

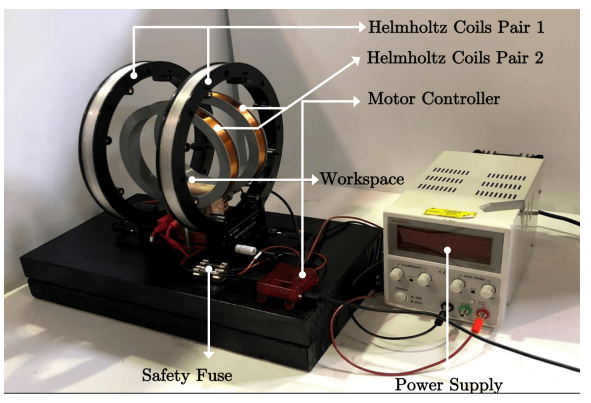

(c) Experimental setup for validation

Fig. 8: Displacement optimization and Turning time results from simulations/experiments and Experimental Setup.

TABLE I: Results from translation, turning in place and trajectory tracking averaged over 5 runs

\begin{tabular}{|l|l|l|l|l|}
\hline Swimmer & $\begin{array}{l}\text { Straight locomotion }(\text { de- } \\
\text { rived from Eq. 15) } \\
{[\mathrm{BL} / \mathrm{s}]}\end{array}$ & $\begin{array}{l}\text { Straight locomotion (de- } \\
\text { rived from Eq. 17) } \\
{[\mathrm{BL} / \mathrm{s}]}\end{array}$ & $\begin{array}{l}\text { Turning (oscillating input) } \\
{[\mathrm{rad} / \mathrm{s}]}\end{array}$ & Turning (constant input) \\
\hline Two-links & $3.663 e-3 \pm .305 e-3$ & $1.4173 e-3 \pm .00331 e-3$ & $5.445 e-1 \pm .468 e-1$ & $1.606 e-1 \pm .0174 e-1$ \\
\hline Three-links & $200.973 e-3 \pm .321 e-3$ & No translation observed & $1.376 e-1 \pm .380 e-1$ & $9.466 e-2 \pm 1.77 e-2$ \\
\hline
\end{tabular}

the magnets at that height. To realize a desired inter-link magnetization ratio, we conduct a turning time test. In this test, we investigate the time a single link submerged in glycerin takes to turn and align itself with constant magnetic field as a function of its internal magnetization. From simulations, we find that the time taken to turn is inversely related to internal magnetization. To verify this experimentally, we magnetize several links at different field strengths, measure the local magnetizing field on the surface of the link with a Gaussmeter at the placement height and measure the time to turn by $\frac{\pi}{2}$ for these links using OpenCV. As Fig. 8b shows, the experimental data matches well with simulations, and indeed this test allows us to compare magnetizations between different links and can be used to fabricate a swimmer with a desired magnetizations. Following this procedure, we fabricated a batch of 12 swimmers with identical magnetization in the first link and varying magnetizations in the second. Each swimmer was actuated with $u(t)=B_{0}(1, \sin 2 \pi f t)$ at $B_{0}=40 G$, $f=0.8 \mathrm{~Hz}$ and time taken to cross between two points at fixed distance was measured, for computing average speed. We conducted five runs per swimmer to compute mean and standard deviation as shown in Fig. 8a. Notice that overall, the experimental result follows the same trend as simulation i.e. average speed increases with magnetization. However, there is a significant difference between the two curves. This can be described as follows: our Neodymium magnet pair setup generates a highly nonuniform magnetic field that varies from $0.1 \mathrm{~T}$ to $0.6 \mathrm{~T}$ in a 0 to $3 \mathrm{~cm}$ long column and the height of a link itself is $0.5 \mathrm{~cm}$. So precise control of numerical values of induced magnetization in a link is difficult using our setup. Secondly, in simulated model, we assumed that the joint between two links is springless. However, for the experimental swimmer, we used an elastomer to connect the links. This elastomer acts like a torsional spring which applies a torque resisting deformation from magnetic torque due to which the swimmer does not undulate as much as in simulations. Links magnetized at weaker fields experience more torsional resistance so the experimental result tends to be further away from simulation. Finally, note that we use normalized units to denote magnetization on $X$ axis in Figs. 8a, 8b because we dont have exact measurements of magnetizations induced in the links. We assume that $M_{\text {induced }} \propto B_{\text {magnetizing }}$ so the $X$ axis for experimental results is indeed the magnetizing field strength in normalized units. For simulation result, we obtained an estimate value of magnetization $0.12 \pm 0.02$ Am by following the magnetizing procedure in [13] for our fabricated links and scaled it proportionately for the 12 swimmers. Hence, our models are slightly approximate because we dont have precise values of internal magnetizations. Controlling and estimating precise values of induced magnetizations in these links is part of future work

\section{EXPERIMENTAL VALIDATION OF MOTION PLANS}

After fabricating the swimmers, we are now ready to experimentally validate motion primitives derived in Section. V. Fig. 8c shows the setup which consists of two pairs of Helmholtz coils connected to a power supply via a Roboclaw motor driver. The strength of the fields produced by these coils is $\sim 40 G$. The size of the workspace is $6 \mathrm{cms} \times 10$ cms. We measured the magnetic field in the workspace on a grid of equally spaced points to check for field gradients and concluded that the field is spatially uniform (see supplementary material for a plot). The swimmer $(L=1.3 \mathrm{cms})$ is fully submerged in glycerin which has a viscosity $1.706 \mathrm{~Pa} . \mathrm{s}$ and density $1260 \mathrm{~kg} / \mathrm{m}^{3}$. In translation, it has average speed close to $0.0004 \mathrm{~m} / \mathrm{s}$. The resulting $R e$ is $\approx 0.0035$ which is well-within the low-Re regime. We now describe the results of translation, turning in place and rectangular trajectory tracking.

\section{A. Translation using classical primitive Eq. 15}

We now present our results from applying $\boldsymbol{u}_{\text {trans }}(t)=$ $B_{0}(1, \sin 2 \pi f t)$ to the two link and three link swimmers. For 


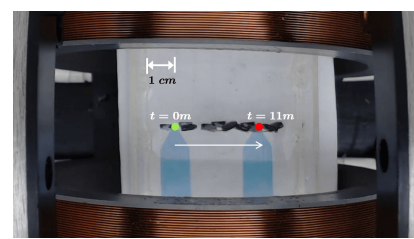

(a) Translation using Eq. 15

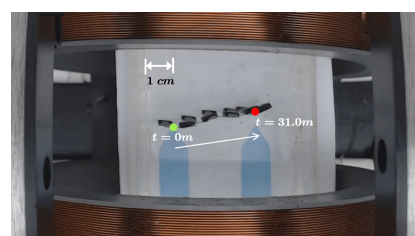

(b) Translation using Eq.17

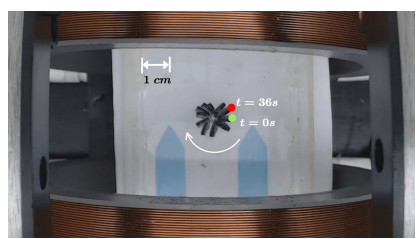

(c) Oscillating input turning

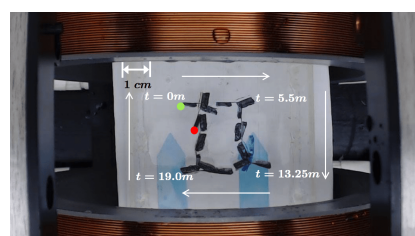

(d) Rectangle tracking

Fig. 9: Results from two-link swimmer (magnetization: 1:6). See supplementary videos for experiments.

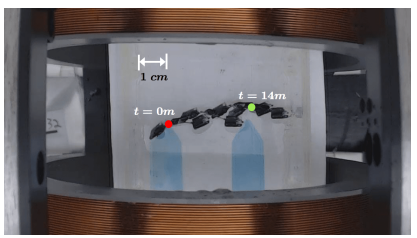

(a) Translation using Eq. 15

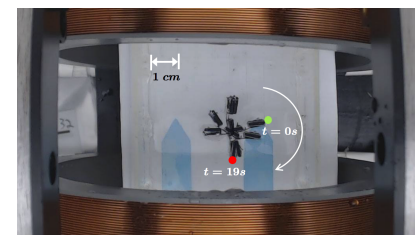

(b) Turning with constant input

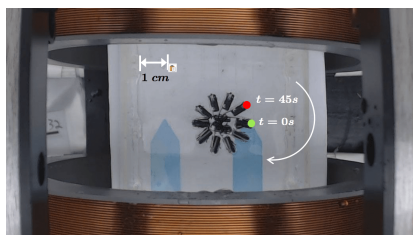

(c) Oscillating input turning

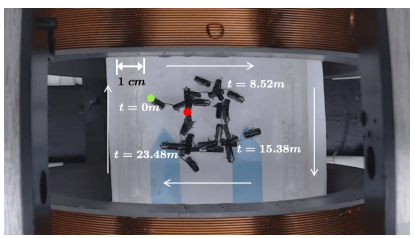

(d) Rectangle tracking

Fig. 10: Results from three-link swimmer (magnetization: 1:4:4). See supplementary videos for these experiments.

these experiments, we used $B_{0}=40$ Gauss and $f=0.8 \mathrm{~Hz}$. Fig. 9a and 10a depicts snapshots of the translation in the two and three link swimmers as a function of time. The average speed can be noted from the second column in Table I.

\section{B. Translation using new primitive Eq. 17}

We also show translation using the new primitive (Eq. 17) derived in Sec.V. Fig. 9b shows snapshots of the two link swimmer with this input. The average speed of the swimmer with this input (third column in Table I) is roughly $40 \%$ of the average speed in the swimmer using the classical primitive, which closely matches with the result from simulations (50\%) (Fig. 5 and Fig. 6). Additionally, from simulations, we obtained that the trajectory from this input is sensitive to the initial orientation of the swimmer. This explains why the trajectory observed in experiments is not fully straight. This input does not result in translation in three-link swimmer (neither in simulations) so we omit that result in Table I.

\section{Turn in place with oscillatory and constant inputs}

We identified that input $\boldsymbol{u}(t)=B_{0}(1, \sin \omega t)$ allows us to switch the direction of magnetic field vector to induce locomotion along a given direction. We can modulate the field in a continuous fashion by composing it with a frequency component slower than the actuating frequency. This causes the swimmer to continuously rotate as the instantaneous magnetic field vector on average also rotates. Fig. 9c and 10c demonstrate an example of this motion. For this experiment, we used $\boldsymbol{u}_{\text {rot }}=R_{\omega_{\text {slow }}} \boldsymbol{u}(t)$ where $\omega=2 \pi f, \omega_{\text {slow }}=\omega / 10$ and $f=0.8 \mathrm{~Hz}$. Alternatively, we can switch and maintain a constant magnetic along a given orientation to induce turning in place (Fig. 10b) (similar to orientation of magnetic compass with external field). The angular velocities for these motions can be compared in the fourth and fifth columns in Table I.

\section{Rectangular trajectory following}

Similarly, we can make the swimmer track a rectangular trajectory by synthesizing switching time instants at which the magnetic field vector turns counter clockwise by $\frac{\pi}{2}$ radians. Whenever this happens, the swimmer turns in place and exhibits a transient response while it turns, which eventually diminishes and steady translation is obtained along the constant component. Fig. 9d and 10d depict snapshots of swimmers following rectangular trajectory using:

$$
\boldsymbol{u}(t)= \begin{cases}\boldsymbol{u}_{\text {trans }}(t) & 0 \mathrm{mins} \leq t \leq 15 \mathrm{mins} \\ R_{\frac{\pi}{2}} \boldsymbol{u}_{\text {trans }}(t) & 15 \mathrm{mins}<t \leq 28 \mathrm{mins} \\ R_{\pi} \boldsymbol{u}_{\text {trans }}(t) & 28 \mathrm{mins}<t \leq 44 \mathrm{mins} \\ R_{\frac{3 \pi}{2}} \boldsymbol{u}_{\text {trans }}(t) & 44 \mathrm{mins}<t \leq 62 \mathrm{mins}\end{cases}
$$

\section{Conclusions}

In this paper, we explored motion planning and efficiency optimization for a two-link magnetic swimmer actuated using magnetic fields. Starting with a mathematical model, we identified a representative class of swimmers and showed how tools from geometric mechanics can be used to synthesize novel motion primitives for this swimmer. We analyzed the efficiency of locomotion using these primitives and further explored how to improve it by optimizing the internal magnetization distributions in the swimmer. We demonstrated an experimental procedure to fabricate these optimal swimmers. Although our results from the model were different from the experimental results, we still have a starting approach for fabricating optimal swimmers through our results. We also conducted experiments to verify translation, trajectory tracking and turn in place motions in two link and three link swimmers. In future, we are interested in resolving the difference in theoretical and simulation results from efficiency analysis, and extending the numerical design optimization and geometric control synthesis tools to multi-link magnetic swimmers.

\section{$X$. AcKnowledgements}

Authors would like to thank Patrick Vedova and Wanyi Chen for help with experiments. This research was supported by the National Science Foundation through the grant CNS-1739308. 


\section{REFERENCES}

[1] F Alouges, Antonio DeSimone, L Giraldi, and M Zoppello. Self-propulsion of slender micro-swimmers by curvature control: N-link swimmers. International Journal of Non-Linear Mechanics, 56:132-141, 2013.

[2] François Alouges, Antonio DeSimone, Laetitia Giraldi, and Marta Zoppello. Can magnetic multilayers propel artificial microswimmers mimicking sperm cells? Soft Robotics, 2(3):117-128, 2015.

[3] François Alouges, Antonio Desimone, Laetitia Giraldi, and Marta Zoppello. Purcell magneto-elastic swimmer controlled by an external magnetic field. IFACPapersOnLine, 50(1):4120-4125, 2017.

[4] Leif E Becker, Stephan A Koehler, and Howard A Stone. On self-propulsion of micro-machines at low Reynolds number: Purcell's three-link swimmer. Journal of fluid mechanics, 490:15-35, 2003.

[5] Andrejs Cebers and Kaspars Erglis. Flexible magnetic filaments and their applications. Advanced Functional Materials, 26(22):3783-3795, 2016.

[6] Hermes Gadêlha. On the optimal shape of magnetic swimmers. Regular and Chaotic Dynamics, 18(1-2):7584, 2013.

[7] Wei Gao, Daniel Kagan, On Shun Pak, Corbin Clawson, Susana Campuzano, Erdembileg Chuluun-Erdene, Erik Shipton, Eric E Fullerton, Liangfang Zhang, Eric Lauga, et al. Cargo-towing fuel-free magnetic nanoswimmers for targeted drug delivery. small, 8(3):460-467, 2012.

[8] Ambarish Ghosh and Peer Fischer. Controlled propulsion of artificial magnetic nanostructured propellers. Nano letters, 9(6):2243-2245, 2009.

[9] Jaskaran Grover, Jake Zimmer, Tony Dear, Matthew Travers, Howie Choset, and Scott David Kelly. Geometric Motion Planning for a Three-Link Swimmer in a Three-Dimensional low Reynolds-Number Regime. In 2018 Annual American Control Conference (ACC), pages 6067-6074. IEEE, 2018.

[10] Emiliya Gutman and Yizhar Or. Simple model of a planar undulating magnetic microswimmer. Physical Review E, 90(1):013012, 2014.

[11] Emiliya Gutman and Yizhar Or. Optimizing an undulating magnetic microswimmer for cargo towing. Physical Review E, 93(6):063105, 2016.

[12] Ross L Hatton and Howie Choset. Geometric motion planning: The local connection, Stokes theorem, and the importance of coordinate choice. The International Journal of Robotics Research, 30(8):988-1014, 2011.

[13] Wenqi Hu, Guo Zhan Lum, Massimo Mastrangeli, and Metin Sitti. Small-scale soft-bodied robot with multimodal locomotion. Nature, 554(7690):81, 2018.

[14] B Jang, E Gutman, N Stucki, BF Seitz, PD Wendel Garcia, T Newton, J Pokki, O Ergeneman, S Pane, Y Or, et al. Undulatory Locomotion of Magnetic Multilink Nanoswimmers. Nano letters, 15(7):4829, 2015.

[15] Scott D Kelly and Richard M Murray. Geometric phases and robotic locomotion. Journal of Field Robotics, 12 (6):417-431, 1995.

[16] Islam SM Khalil, Herman C Dijkslag, Leon Abelmann, and Sarthak Misra. MagnetoSperm: A microrobot that navigates using weak magnetic fields. Applied Physics Letters, 104(22):223701, 2014.

[17] Kathrin E Peyer, Li Zhang, and Bradley J Nelson. Bioinspired magnetic swimming microrobots for biomedical applications. Nanoscale, 5(4):1259-1272, 2013.

[18] Edward M Purcell. Life at low Reynolds number. Am. J. Phys, 45(1):3-11, 1977.

[19] Masahiko Sendoh, Kazushi Ishiyama, and K-I Arai. Fabrication of magnetic actuator for use in a capsule endoscope. IEEE Transactions on Magnetics, 39(5): 3232-3234, 2003.

[20] Daniel Tam and Annete E Hosoi. Optimal stroke patterns for Purcells three-link swimmer. Physical Review Letters, 98(6):068105, 2007.

[21] Oren Wiezel and Yizhar Or. Optimization and smallamplitude analysis of Purcell's three-link microswimmer model. arXiv preprint arXiv:1603.02268, 2016. 